\title{
Promotion of astrocytoma cell invasion by micro RNA-22 targeting of tissue inhibitor of matrix metalloproteinase-2
}

\author{
Yu-ichiro Ohnishi, MD, PhD, ${ }^{1}$ Koichi Iwatsuki, MD, PhD, ${ }^{1}$ Masahiro Ishihara, MD, PhD, ${ }^{1}$ \\ Toshika Ohkawa, MD, PhD, ${ }^{2}$ Manabu Kinoshita, MD, PhD, ${ }^{3}$ Koei Shinzawa, PhD, ${ }^{4}$ \\ Yasunori Fujimoto, MD, PhD, and Toshiki Yoshimine, MD, PhD ${ }^{1}$
}

\begin{abstract}
Departments of ${ }^{1}$ Neurosurgery and ${ }^{4}$ Molecular Genetics, Osaka University Medical School; ${ }^{2}$ Department of Neurosurgery, Yao Municipal Hospital; and '3epartment of Neurosurgery, Osaka Medical Center for Cancer and Cardiovascular Diseases, Osaka, Japan
\end{abstract}

OBJECTIVE Diffuse astrocytomas (DAs) have a high recurrence rate due to diffuse infiltration into the brain and spinal cord. Micro RNAs (miRNAs) are small noncoding RNAs that regulate gene expression by binding to complementary sequences of target messenger RNA (mRNA). It has been reported that miRNA-22 (miR-22) is involved in the invasion of some cancer cell lines. The aim of this study was to identify the biological effects of miR-22 in regard to the invasion of human DAs.

METHODS The authors evaluated whether the level of miR-22 is elevated in human spinal DAs by using miRNA chips. Next, the role of miR-22 in 1321N1 human astrocytoma cells was investigated. Finally, to elucidate whether miR-22 promotes invasion by astrocytoma cells in vivo, the authors transplanted miR-22 overexpressed astrocytoma cells into mouse thoracic spinal cord.

RESULTS The miR-22 significantly upregulated the invasion capacity of $1321 \mathrm{~N} 1 \mathrm{cells}$. Computational in silico analysis predicted that tissue inhibitor of matrix metalloproteinase-2 (TIMP2) is a target gene of miR-22. This was confirmed by quantitative reverse transcription polymerase chain reaction and Western blotting, which showed that miR-22 inhibited TIMP2 mRNA and protein expression, respectively. Luciferase reporter assays demonstrated that miR-22 directly bound the 3'-untranslated regions of TIMP2. The authors further showed that miR-22 promoted invasiveness in 1321 N1 astrocytoma cells when transplanted into mouse spinal cord.

CONCLUSIONS These data suggest that miR-22 acts to regulate invasion of $1321 \mathrm{~N} 1$ astrocytoma cells by targeting TIMP2 expression. Additional studies with more cases and cell lines are required to elucidate the findings of this study for a novel treatment target for spinal DAs.

https://thejns.org/doi/abs/10.3171/2016.8.SPINE16248

KEY WORDS astrocytoma; invasion; micro RNA-22; miR-22; spinal cord; tissue inhibitor of matrix metalloproteinase-2; TIMP2

$\mathrm{D}$ IFFUSE astrocytomas (DAs) are slow-growing tumors, and patients with DAs have a median survival ranging from 5 to 8 years. These tumors have a high recurrence rate because of diffuse infiltration to surrounding brain and spinal cord tissues, and an inherent malignant potential to transform into anaplastic astrocytomas and glioblastomas. ${ }^{11,18}$ The main therapy for DAs remains controversial, but the effects of radiation and chemotherapy have been reported. ${ }^{1}$
Spinal astrocytoma is rare. The intramedullary types account for $1 \%-2 \%$ of CNS tumors, $40 \%$ of which involve astrocytomas. ${ }^{3,22}$ Spinal astrocytomas are considered to be histopathologically equal to brain astrocytomas. Some spinal astrocytomas have a dissection plane between the tumor and the healthy spinal cord, which may allow total resection. However, many spinal astrocytomas have no dissection plane, in which case total resection may be difficult to achieve without injuring the spinal cord and causing

ABBREVIATIONS DA = diffuse astrocytoma; $E C M=$ extracellular matrix; miRNA, miR-22, mRNA = micro RNA, micro RNA-22, messenger RNA; MMP = matrix metalloproteinase; PFA = paraformaldehyde; RT-PCR = reverse transcription polymerase chain reaction; TBST = Tris-buffered saline with Triton X-100; TIMP2 = tissue inhibitor of matrix metalloproteinase-2; 3'-UTR = 3'-untranslated region.

SUBMITTED March 1, 2016. ACCEPTED August 3, 2016.

INCLUDE WHEN CITING Published online November 11, 2016; DOI: 10.3171/2016.8.SPINE16248. 
neurological deterioration. ${ }^{6,10,11,15,18,22}$ Therefore, the treatment of spinal astrocytomas demands adjunctive measures to suppress the invasion.

Micro RNAs (miRNAs) are small noncoding RNAs that regulate gene expression by binding to complementary sequences in the $3^{\prime}$-untranslated regions (3'-UTRs) of target messenger RNA (mRNA), thereby inducing degradation and suppressing translation of target mRNA. ${ }^{8}$ The miRNAs regulate various target genes, including oncogenes and tumor suppressor genes. ${ }^{13}$ Deregulation of miRNA-22 (miR-22) has been observed in various cancers. It has been reported that miR-22 is implicated in the regulation of various cellular processes, including cell growth, apoptosis, and the cell cycle, $, 2,26,27,29,32$ and it is also involved in the invasion of some cancer cell types. ${ }^{12,28}$ However, we lack a detailed understanding of its role in DAs.

In this study, our aim was to identify the biological effects of miR-22 in regard to the invasion of human DAs, especially in the spinal cord tissue. First, we evaluated whether the level of miR-22 is elevated in human spinal DAs by using miRNA chips. Next, we investigated the role of miR-22 in astrocytoma cells. Finally, to elucidate whether miR-22 promotes invasion by astrocytoma cells in vivo, we transplanted miR-22 overexpressed astrocytoma cells into mouse thoracic spinal cord.

\section{Methods}

\section{Experimental Design}

This study was approved by the Ethics Committee of the Osaka University Medical School. All procedures on patients were performed after obtaining written, informed consent. Animal experiments were performed in accordance with the guidelines of the Laboratory Animals Care and Use Committee of the Osaka University Faculty of Medicine. Every effort was made to limit the number of animals used and to minimize their suffering.

\section{Extraction of RNA and Profiling of miRNA Expression}

Sections $(10 \mu \mathrm{m})$ were prepared from each formalinfixed paraffin-embedded specimen. The specimens were obtained from spinal DAs removed in the patients in Cases 11,12 , and 13 by subtotal or total resection. Paraffin was removed by xylene treatment, and then tissues were washed with ethanol twice to remove the xylene. Tissues were then treated with proteinase $\mathrm{K}$ at $37^{\circ} \mathrm{C}$ overnight. Following centrifugation, the supernatant was processed using a silica-based spin column (Toray Industries) to obtain purified total RNA. The degree of RNA cross-linking and RNA degradation was analyzed by electrophoresis, in which an Agilent 2100 Bioanalyzer (Agilent Technologies) was used. The concentration of the RNA solution was determined by measuring its absorbance at $260 \mathrm{~nm}$.

Extracted total RNA was labeled with Hy5, using a miRCURY LNA Array miRNA labeling kit (Exiqon). Labeled RNAs were hybridized onto 3D-Gene Human miRNA Oligo chips (Toray Industries). The annotation and oligonucleotide sequences of the probes conformed to the miRBase miRNA database (http://microrna.sanger.ac.uk/ sequences/). After stringent washing, fluorescent signals were scanned using a 3D-Gene Scanner (Toray Industries) and analyzed using 3D-Gene Extraction software (Toray Industries). ${ }^{24,25}$

The raw data from each spot were normalized by substitution with the mean intensity of the background signal, which was determined from the signal intensities of all the blank spots at a 95\% confidence interval. Measurements from spots whose signal intensities were $>2$ SDs from the background signal intensity were considered to be valid. The relative expression level of a given miRNA was calculated by comparing the signal intensities of the valid spots throughout the microarray experiment. The data were globally normalized per array, such that the median of the signal intensity was adjusted to 25 .

\section{Cell Lines}

The 1321N1 astrocytoma cells were cultured in DMEM (Life Technologies) supplemented with $100 \mathrm{U} / \mathrm{ml}$ penicillin (Wako Kyoto), $100 \mu \mathrm{g} / \mathrm{ml}$ streptomycin (Wako), and $10 \%$ (vol/vol) fetal bovine serum (Biowest) at $37^{\circ} \mathrm{C}$ in a humidified incubator with $5 \% \mathrm{CO}_{2} .{ }^{16}$ The cells were passaged using $0.25 \%$ trypsin-EDTA (Thermo Fisher Scientific).

\section{Extraction of RNA and Performance of Quantitative Real-Time Polymerase Chain Reaction}

Total RNAs and miRNAs were extracted using a mirVana miRNA isolation kit according to the manufacturer's instructions. Reverse transcription polymerase chain reaction (RT-PCR) was performed using a SuperScript VILO cDNA synthesis kit (Invitrogen). The expression of TIMP2 was assayed using TaqMan array plates (Thermo Fisher Scientific). We used GAPDH (glyceraldehyde3-phosphate dehydrogenase), 18S ribosome, HPRT1, and GUSB as endogenous references. Gene expression was detected by real-time PCR performed using a ViiA 7 realtime PCR system (Thermo Fisher Scientific), and miRNA expression was determined using the TaqMan MicroRNA assay and the same PCR system, in accordance with manufacturer's instructions. We used U6 as an endogenous reference. Independent experiments were repeated 3 times for each sample. The relative expression levels of mRNA and miRNA were analyzed using the $2^{-\Delta \Delta \mathrm{Ct}}$ method.

\section{Transfection Protocol}

Cells were seeded in a 24 -well plate $\left(10^{5}\right.$ per well), and cultured for 24 hours. The miRNA mimic, miRNA inhibitor, mimic control miRNA, and inhibitor control miRNA (mirVana miRNA mimics, inhibitors, and control; Thermo Fisher Scientific) or expression plasmids were transfected into the cells by using Lipofectamine 2000 (Life Technologies) according to the manufacturer's protocol. The cells were cultured in a humidified atmosphere containing $5 \% \mathrm{CO}_{2}$ at $37^{\circ} \mathrm{C}$ for 6 hours, and then the transfection medium was replaced with complete medium. These cells were used for various assays following incubation for 48 hours.

\section{Luciferase Reporter Assay}

The full-length 3'-UTR of TIMP2 was cloned into the psiCHECK2 luciferase reporter vector (Promega). Renilla 
luciferase was used as the primary reporter gene, and wildtype or mutant-type 3'-UTR of TIMP2 was cloned into a multiple cloning region located downstream of the Renilla luciferase translational stop codon. The psiCHECK-2 vector contains a second reporter gene, firefly luciferase, which allows normalization of Renilla luciferase expression.

A PrimeSTAR mutagenesis basal kit (Takara Bio, Inc.) was used to generate a mutant 3'-UTR of TIMP2 in which UGU is substituted with CAC in the miR-22-binding region. In accordance with the manufacturer's instructions, Lipofectamine 2000 was used to cotransfect the miR-22 mimic by using psiCHECK 2 vector containing either the wild-type or mutant-type 3'-UTR of TIMP2 into 1321N1 cells. After incubation in a humidified atmosphere containing $5 \% \mathrm{CO}_{2}$ at $37^{\circ} \mathrm{C}$ for 48 hours, a luciferase reporter assay was performed using a dual-luciferase reporter assay system (Promega) according to the manufacturer's instructions. Each assay was performed in triplicate. Renilla luciferase was used for normalization.

\section{Western Blotting}

Western blotting was performed as previously described. ${ }^{21}$ Briefly, total protein was extracted from 1321N1 cells by using extraction buffer (10 mM Tris-HCl [Nippon Gene], pH 7.4), 0.1\% sodium dodecyl sulfate (Nippon Gene), $1 \%$ Triton $X$ (Sigma-Aldrich), and $100 \mathrm{mM} \mathrm{NaCl}$ (Wako) supplemented with protease inhibitors (Halt protease inhibitor cocktail, Thermo Scientific) and phenylmethanesulfonyl fluoride (Sigma-Aldrich). Protein concentrations were measured with a Qubit protein assay kit (Invitrogen) after microcentrifugation.

Samples were resolved by electrophoresis on NuPAGE Novex 4\%-12\% bis-Tris gels (Invitrogen) and transferred to polyvinylidene fluoride membranes (Invitrogen). Blots were blocked with 5\% ECL blocking agent (GE Healthcare Life Sciences) in Tris-buffered saline with Triton X-100 (TBST) (Nippon Gene) for 1 hour, and then incubated with the primary antibody (anti-TIMP2 [1:2000, rabbit; Abcam] and anti- $\alpha$-tubulin [1:5000, mouse; Abcam]) in TBST overnight at $4^{\circ} \mathrm{C}$. After washing with TBST, membranes were incubated with horseradish peroxidase-conjugated antirabbit (1:2000; Cell Signaling Technology) or horseradish peroxidase-conjugated antimouse secondary antibody (1:50,000; GE Healthcare Life Sciences) for 1 hour at room temperature. Blots were washed again with TBST and developed with ECL Plus reagent (GE Healthcare Life Sciences). Signal intensities were determined using an IS-8000 FluorChem digital imaging system (Alpha Innotech). Data were analyzed by densitometry performed using ImageJ, and normalized to $\alpha$-tubulin.

\section{Invasion Assay}

A CytoSelect 24-well cell invasion assay kit (Cell Biolabs, Inc.) was used to explore the invasion capacity of 1321N1 cells. Following the manufacturer's instructions, cells were suspended in serum-free DMEM to $5 \times 10^{5}$ cells/ $\mathrm{ml}$. For the invasion assay, $500 \mu \mathrm{l}$ of DMEM with $10 \%$ fetal bovine serum was added to the lower chamber and 300 $\mu l$ of cell suspension was added to the upper chamber. The upper surface of the insert membrane is coated with a uni- form layer of dried basement membrane matrix solution. After incubation in a humidified atmosphere containing $5 \% \mathrm{CO}_{2}$ at $37^{\circ} \mathrm{C}$ for 48 hours, noninvaded cells and media were gently removed from the inserts using aspiration and cotton-tipped swabs. Each insert was transferred to an empty well in a plate, $200 \mu \mathrm{l}$ of extraction solution was added per well, and then the sample was incubated for 10 minutes on an orbital shaker. The invaded cells attached to the lower surface of the membrane inserts were stained and quantified by transferring $100 \mu \mathrm{l}$ of each sample to a 96-well microtiter plate. Optical densities resulting from the stained invasive cells were determined at $\lambda 560 \mathrm{~nm}$ with the aid of a plate reader.

\section{Transplantation Into Mouse Spinal Cord}

The pCMV-MIR22 vector expresses the precursor of miR-22 and GFP (OriGene), and the pCMV-MIR vector is a control empty vector that expresses GFP alone (OriGene). In accordance with the manufacturer's instructions, Lipofectamine 2000 was used to transfect pCMVMIR22 or pCMV-MIR empty vector into 1321N1 cells. The cells were collected after incubation in a humidified atmosphere containing $5 \% \mathrm{CO}_{2}$ at $37^{\circ} \mathrm{C}$ for 48 hours.

Seven adult male C57BL/6 J mice (8-9 weeks old; Japan SLC, Inc.) were given inhalation anesthesia with sevoflurane (1.5\%; Maruishi Pharmaceutical) and $\mathrm{O}_{2}$. After laminectomy at the 10th thoracic spinal vertebra, the dorsal surface of the dura mater was exposed. The cells were transplanted into the mice by using a Hamilton syringe connected to a 33-gauge needle, aided by a stereotaxic injector (Narishige). The cells for transplantation were trypsinized and diluted in serum-free DMEM to $5 \times 10^{5}$ cells/ $\mu \mathrm{l}$ (control vector, $\mathrm{n}=3$; miR-22 expression vector, $\mathrm{n}$ $=4)$. The needle was inserted $1 \mathrm{~mm}$ into the spinal cord through the dorsal midline to avoid injury of the posteromedian medullary vein. Cell suspension $(2 \mu \mathrm{l})$ was injected at $1 \mu \mathrm{l} /$ minute, and then 1 minute after the completion of delivery the needle was retracted. All the mice received daily subcutaneous injections of FK506 (5 mg/ $\mathrm{kg})$ as an immunosuppressant for 1 week after transplantation.

One week posttransplantation, the mice were killed with a lethal dose of pentobarbital sodium, and the spinal cords were fixed by transcardial perfusion with $100 \mathrm{ml}$ phosphate-buffered saline followed by $4 \%$ paraformaldehyde (PFA). Spinal cords were fixed in $4 \%$ PFA overnight, cryoprotected in $30 \%$ sucrose, embedded in OCT compound (Tissue Tek, Sakura Finetek), and frozen. Sections (10 $\mu \mathrm{m}$ thick) were sagittally cut from the blocks using a cryostat (CM1510S; Leica).

\section{Immunocytochemical Analysis}

Immunocytochemistry was performed as previously described. ${ }^{19-21}$ Cells were fixed with $4 \%$ PFA, incubated for 1 hour at room temperature in blocking solution, incubated overnight at $4^{\circ} \mathrm{C}$ with the primary antibody, washed, and then incubated overnight at $4^{\circ} \mathrm{C}$ with the appropriate species-directed secondary antibody. The primary antibodies were anti-Ki 67 (1:200 mouse monoclonal antibody; Abcam), anti-TIMP2 (1:100, rabbit; Abcam), anti- $\alpha$-tubulin (1:200, mouse; Abcam), and anti-GFP (1:500 goat poly- 
clonal antibody; Abcam). The secondary antibodies used were DyLight 488-conjugated goat anti-mouse (1:200; Kirkegaard and Perry Laboratories) or DyLight 549-conjugated goat anti-rabbit (1:200; Kirkegaard and Perry Laboratories). To visualize cell nuclei, slides were counterstained with 4',6-diamidino-2-phenylindole dihydrochloride (DAPI; Vector Laboratories). The cell phenotype was assessed and cell counts were obtained from images captured with a confocal laser fluorescence microscope (FV-1000D; Olympus). The percentages of Ki 67-positive cells are shown as the mean \pm SD of 3 images from 3 independent experiments. The GFP-positive and $\alpha$-tubulin-positive cell counts were estimated by counting GFPpositive and $\alpha$-tubulin-positive cells, respectively, per field on digitized images. Transfection efficiencies were calculated as $100 \times$ (number of GFP-positive cells)/(number of $\alpha$-tubulin-positive cells).

\section{Immunohistochemical Analysis}

Immunohistochemistry was performed as previously described. ${ }^{19,21}$ Briefly, sections were stained with anti-GFP (1:500 goat polyclonal antibody; Abcam). The secondary antibody used was DyLight 488-conjugated donkey antigoat antibody (1:200; Abcam). The slides were counterstained with DAPI. Sections were assessed from images captured using the confocal laser fluorescence microscope. Three fields per chosen sagittal section were assessed to confirm GFP-positive cells in both groups. We measured the length from the rostral to the caudal position.

\section{Statistical Analysis}

All data are represented as the mean of at least triplicate samples \pm SD. Statistical analysis included 1-way ANOVA or Student t-test using StateMateV, and $\mathrm{p}$ values $<0.05$ were considered statistically significant.

\section{Results}

\section{Promotion of 1321N1 Cell Invasion In Vitro by miR-22}

Five patients underwent surgical treatment for spinal DAs between January 2010 and May 2013 (1 man and 4 women, 37-86 years of age, mean age 52.2 years). We numbered DA cases from 11 to 15 to anonymize. We tried to obtain total RNA from each formalin-fixed paraffinembedded specimen of DA obtained in Cases 11-15 by subtotal or total resection. The RNA obtained from spinal DA in Cases 14 and 15 was degraded. Therefore we have analyzed the DA specimens only in Cases 11, 12, and 13.

The miR-22 expression levels in 3 spinal DA cases (1113) were measured using Toray $3 \mathrm{D}-\mathrm{Gene}$ human miRNA chips to evaluate whether miR-22 is elevated in human spinal DAs. This microarray analysis showed that miR-22 was upregulated in 2 of the 3 spinal DA samples (Fig. 1A).

The role of miR-22 in astrocytoma cells was investigated by transfecting $1321 \mathrm{~N} 1$ cells with miR-22 mimic, miR-22 inhibitor, mimic control, or inhibitor control. The miR-22 mimic is chemically modified double-stranded RNA that mimics endogenous miR-22 and enables miR-22 functional analysis by upregulation of miR-22 activity. The miR-22 inhibitor is chemically modified single-stranded
RNA designed to specifically bind to and inhibit endogenous miR-22, and also to enable miR-22 functional analysis by downregulation of miR-22 activity. Mimic control is designed to be used as a negative control for experiments in which miR-22 mimic is used, and has random sequences. Inhibitor control is intended for use as a negative control for experiments in which miR-22 inhibitor is used, and has random sequences. Quantitative RT-PCR revealed that the expression of miR-22 in the miR-22 mimic group increased approximately 50 -fold compared with that of the nontransfected group (Fig. 1B). The expression of miR-22 in the miR-22 inhibitor group decreased to approximately one-fifth that of the nontransfected group (Fig. 1C).

We determined whether miR-22 is involved in the proliferation of astrocytoma cells by conducting a Ki 67-labeling analysis of transfected $1321 \mathrm{~N} 1$ cells. The percentages of Ki 67-positive cells were not significant among these groups (Fig. 1D). The effect of miR-22 on the invasion capabilities of astrocytoma cells was determined by invasion assays for transfected $1321 \mathrm{~N} 1$ cells. The miR-22 significantly promoted the in vitro invasion capabilities of $1321 N 1$ cells (Fig. 1E and F). Taken together, these findings indicate that miR-22 promoted $1321 \mathrm{~N} 1$ cell invasion, but not proliferation in vitro. Therefore, we explored the target mRNA of miR-22 to elucidate how miR-22 regulates invasion by $1321 \mathrm{~N} 1$ cells.

\section{Direct Targeting of TIMP2 by miR-22}

Bioinformatics analysis showed that TIMP2 is a putative target of miR-22 (Fig. 2A). We used a consensus approach with 2 widely used types of software (miRanda and TargetScan), and speculated that TIMP2 might be involved in miR-22-mediated invasion by $1321 \mathrm{~N} 1$ cells. TIMP2 is a natural inhibitor of the matrix metalloproteinases (MMPs), a group of peptidases involved in degradation of the extracellular matrix (ECM). To verify whether miR-22 directly targeted TIMP2, luciferase reporter assays were conducted. We constructed plasmid with the full-length 3'-UTR of the TIMP2 insert and plasmid with a mutant 3'-UTR of TIMP2, which carried a substitution of 4 nucleotides within the miR-22 binding site. The results of a luciferase reporter assay are shown in Fig. 2B, and indicate that $1321 \mathrm{~N} 1$ cells cotransfected with miR-22 mimic and with wild-type 3 '-UTR of TIMP2 showed a notable decrease in luciferase activity compared with the control group $(\mathrm{p}<0.05)$. However, $1321 \mathrm{~N} 1$ cells cotransfected with miR-22 mimic and with mutant type $3^{\prime}$-UTR of TIMP2 had the same luciferase activity as the control group. These results indicate that TIMP2 is a novel target of miR-22 in astrocytoma cells.

We further examined the expression of TIMP2 in $1321 \mathrm{~N} 1$ cells by transfecting $1321 \mathrm{~N} 1$ cells with miR-22 mimic or miR-22 inhibitor. As shown in Fig. 2C, the miR22 mimic had a negative effect on the TIMP2 expression level compared with the internal controls $(\mathrm{p}<0.05)$, whereas the miR-22 inhibitor had a positive effect compared with the internal controls $(\mathrm{p}<0.05)$.

The effect of miR-22 on TIMP2 protein expression was investigated by transfecting $1321 \mathrm{~N} 1$ cells with miR-22 mimic and mimic control, miR-22 inhibitor and inhibitor control, and then examining the level of TIMP2 protein in miR-22-overexpressing $1321 \mathrm{~N} 1$ cells. As shown in 
A

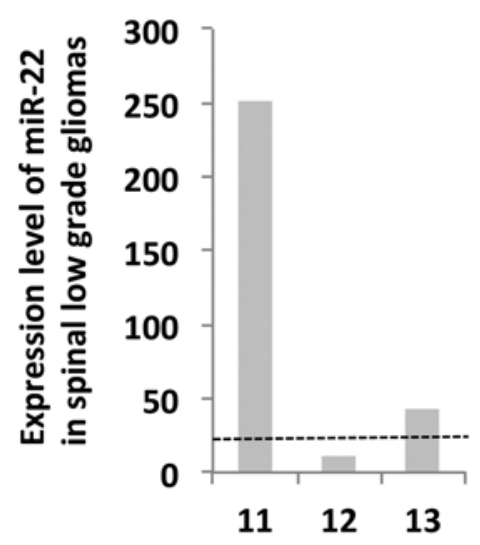

D

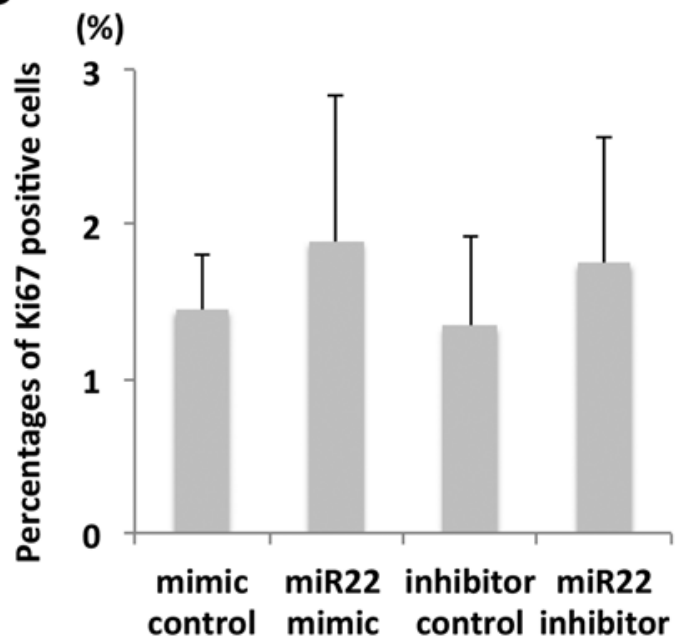

B

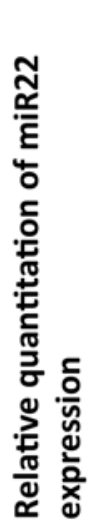

C

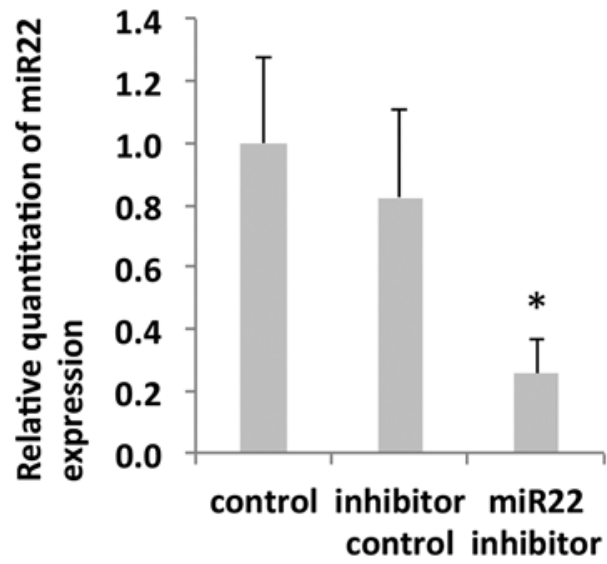

$\mathbf{E}$

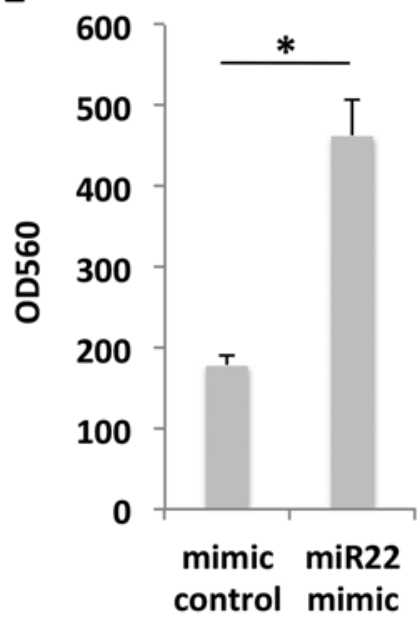

$\mathbf{F}$

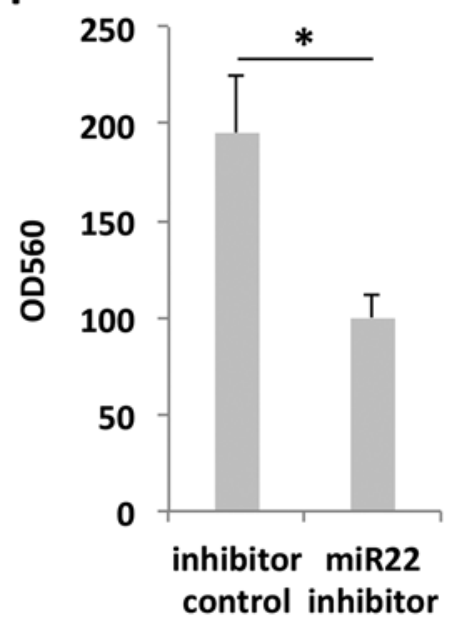

FIG. 1. Bar graphs showing that miR-22 promoted $1321 \mathrm{~N} 1$ cell invasion in vitro. A: Expression level of miR-22 in 3 patients with spinal DA (Cases 11-13). Dashed line indicates the median of the signal intensity. B: Expression of miR-22 in 1321N1 cells not transfected and transfected with miR-22 mimic or control mimic was detected by quantitative RT-PCR. C: Expression of miR-22 in 1321N1 cells not transfected and transfected with miR-22 inhibitor or control inhibitor was detected by quantitative RT-PCR. D: Cell proliferation of 1321N1 cells transfected with miR-22 mimic, control mimic, miR-22 inhibitor, or control inhibitor was detected by Ki $67-l a b e l i n g$ analysis. $\mathrm{E}$ and $\mathrm{F}$ : In the invasion assay, invasive cells on the bottom of the invasion membrane were stained. Optical densities (ODs) resulting from the stained and extracted invasive cells were determined at $\lambda 560 \mathrm{~nm}$ by using a plate reader. For this assessment we used an in vitro invasion assay of $1321 \mathrm{~N} 1$ cells transfected with miR-22 mimic and control mimic, or miR-22 inhibitor and control inhibitor. The miR-22 mimic is chemically modified double-stranded RNA that mimics endogenous miR-22. The miR-22 inhibitor is chemically modified single-stranded RNA designed to specifically bind to and inhibit endogenous miR-22. Control mimic and inhibitor are designed to be used as a negative control for experiments using miR-22 mimic and inhibitor, respectively. ${ }^{*} p<0.05$.

Fig. 2D, the level of TIMP2 protein was downregulated in miR-22-overexpressing $1321 \mathrm{~N} 1$ cells compared with the control group $(\mathrm{p}<0.05)$, and upregulated in miR-22 inhibitor-expressing $1321 \mathrm{~N} 1$ cells compared with the control group. We confirmed that miR-22 negatively regulates the expression of TIMP2 in astrocytoma cells.

\section{Promotion of 1321N1 Cell Invasion In Vivo by miR-22}

To elucidate whether miR-22 promotes invasion by astrocytoma cells in vivo, we transplanted miR-22-overexpressing $1321 \mathrm{~N} 1$ cells into mouse thoracic spinal cord. Immunocytochemical analysis showed that both the control and miR-22 expression vector-transfected cells were pos- itive for $\alpha$-tubulin and GFP. The transfection efficiency in the control and miR-22 expression vector-transfected $1321 \mathrm{~N} 1$ cells was $71.1 \% \pm 21.9 \%$ and $82.3 \% \pm 16.8 \%$, respectively, which are not statistically different $(\mathrm{p}>0.05)$. However, the expression of TIMP2 was downregulated in the miR-22 expression vector group compared with the control group (Fig. 3A).

Sagittal sections of spinal cord obtained during immunohistochemical analysis confirmed the presence of GFPpositive cells in both groups 1 week after transplantation (Fig. 3B and C). The length from the rostral to the caudal sites was measured because the grafted GFP-positive cells were invaded. The extent of GFP-positive grafted cells in 
A

3' a AGAAGUUGACCGUCGA a 5' miR-22 \| | | |||||| $\mid$

5' a GAGCCGGGTGGCAGCT g 3' normal III | | | ||

5' a GAGCCGGGTCCCTCCT g 3' mismatch
B

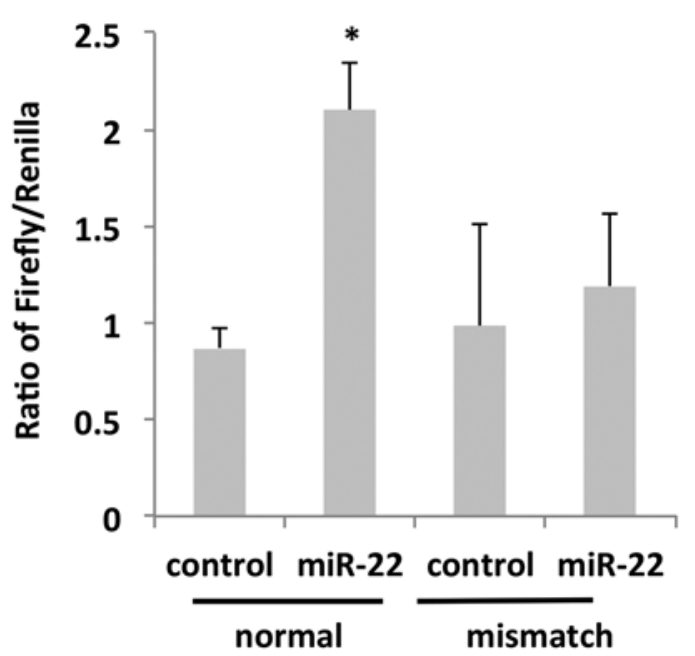

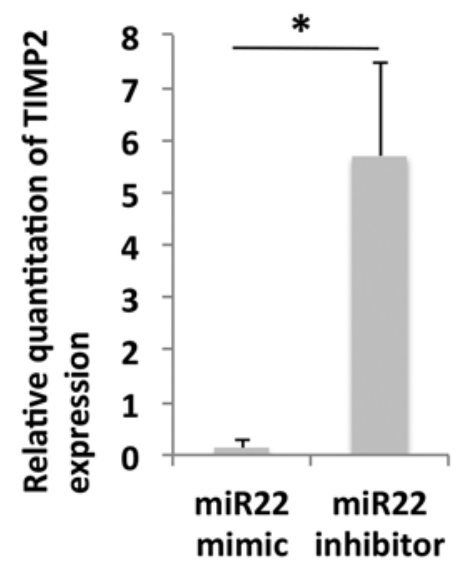

D
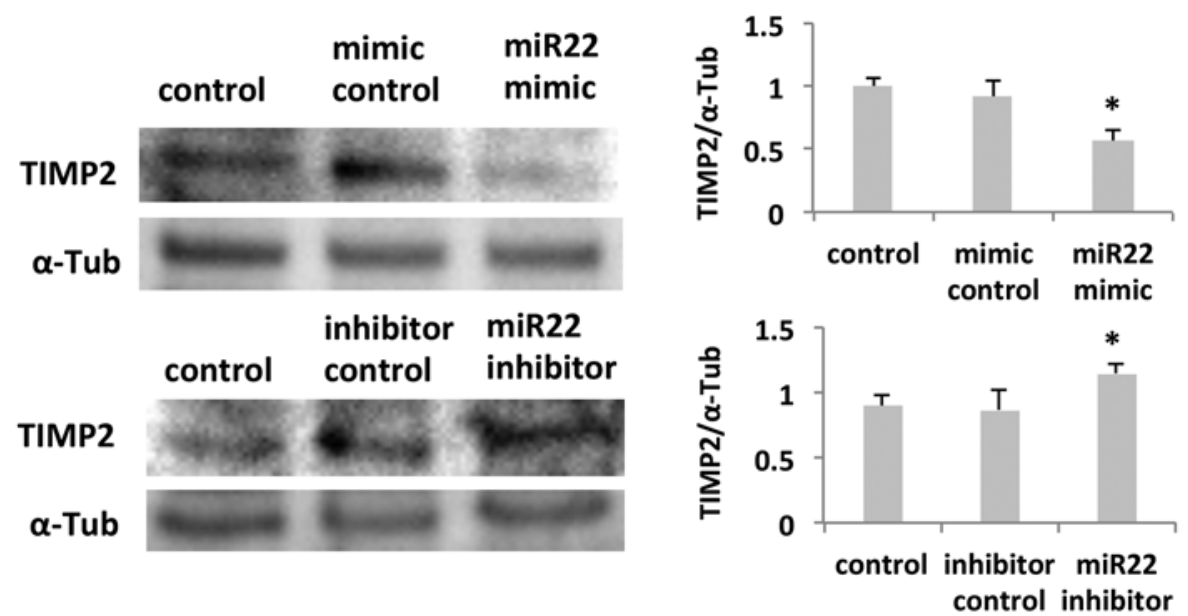

FIG. 2. Bar graphs and Western blots showing that TIMP2 was a direct target of miR-22. A: Data of miRanda and TargetScan showed the putative target sequence of miR-22 in the 3'-UTR of TIMP2. Plasmid with the full-length 3'-UTR of the TIMP2 insert and plasmid with a mutant 3'-UTR of TIMP2, which carried a substitution of 4 nucleotides within the miR-22 binding site, were constructed. B: The 1321N1 cells were cotransfected with miR-22 mimic or control mimic with normal or mismatch TIMP2 3'-UTR. Luciferase activity was assayed. C: Expression of TIMP2 in 1321N1 cells transfected with miR-22 mimic or miR-22 inhibitor was detected by quantitative RT-PCR. D: The protein level in 1321N1 cells not transfected and transfected with miR-22 mimic and inhibitor or control mimic and inhibitor was detected by Western blotting. Data are presented as the mean \pm SD from at least 3 independent experiments. ${ }^{*} p<0.05 . \alpha$-Tub $=\alpha$-tubulin.

the control and miR-22 expression vector group was 57.0 $\pm 51.4 \mu \mathrm{m}$ and $1041.7 \pm 181.6 \mu \mathrm{m}$, respectively (Fig. 3D), indicating that miR-22 promoted invasion by $1321 \mathrm{~N} 1$ cells in vivo.

\section{Discussion}

The present study showed that TIMP2 is a novel molecular target of miR-22 in invasion of 1321N1 astrocytoma cells. We demonstrated that overexpression of miR-22 could promote invasion of $1321 \mathrm{~N} 1$ astrocytoma cells by negatively regulating TIMP2 expression posttranscriptionally via binding to the 3 '-UTR of TIMP2. The miR-22 also promoted invasion of $1321 \mathrm{~N} 1$ astrocytoma cells when transplanted into mouse spinal cord.

Total resection is the main therapy for spinal DA, but radical surgery is difficult because of diffuse infiltra- tion to surrounding spinal cord tissues. Spinal DAs and brain DAs are believed to have the same histopathological characteristics and thus can be treated in the same manner. However, it remains unknown whether the molecular characteristics of spinal DAs correspond to those of brain DAs. Thus, it is important to elucidate the molecular characteristics of spinal DAs to identify adjunctive therapies for countering their invasion.

Degradation of the ECM around tumor cells is an essential step in the process of tumor invasion. Various proteinases participate in the degradation of ECM, and a family of MMPs plays an important role in matrix degradation around tumor cells. ${ }^{4,23}$ A significant correlation between the malignancy of glioma and MMPs has been reported..$^{17}$ The activities of MMPs are strictly regulated by TIMPs, ${ }^{30}$ which comprise 2 structural domains. The $\mathrm{N}$-terminal domain binds to the active site of mature MMPs, blocking 
A
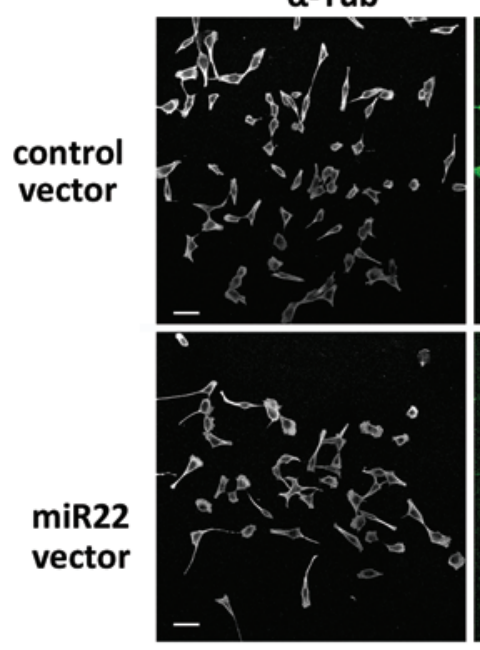

GFP
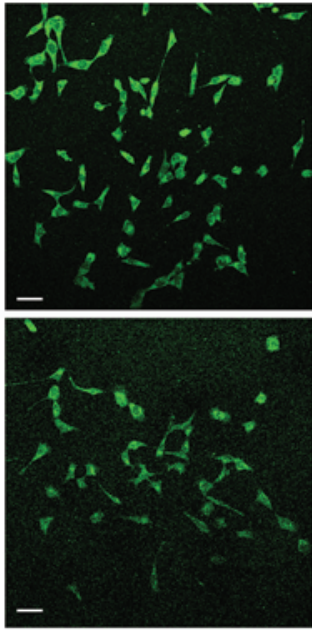

TIMP2

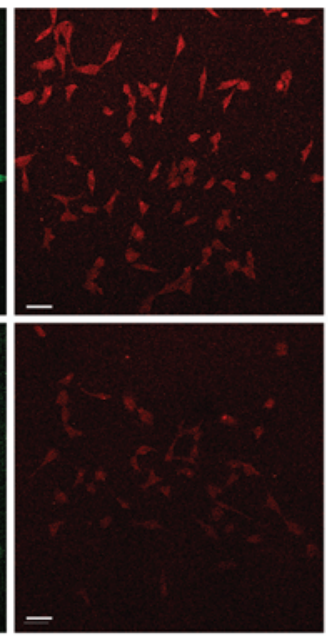

D

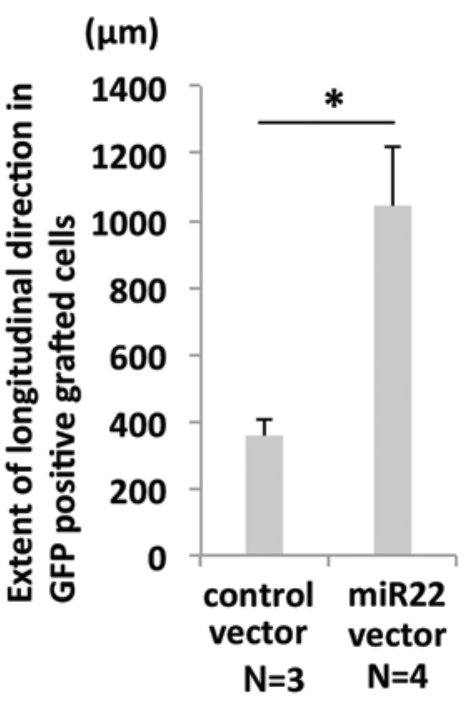

B

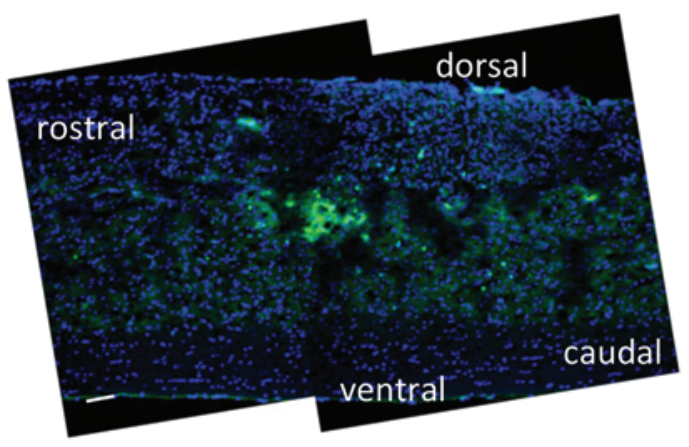

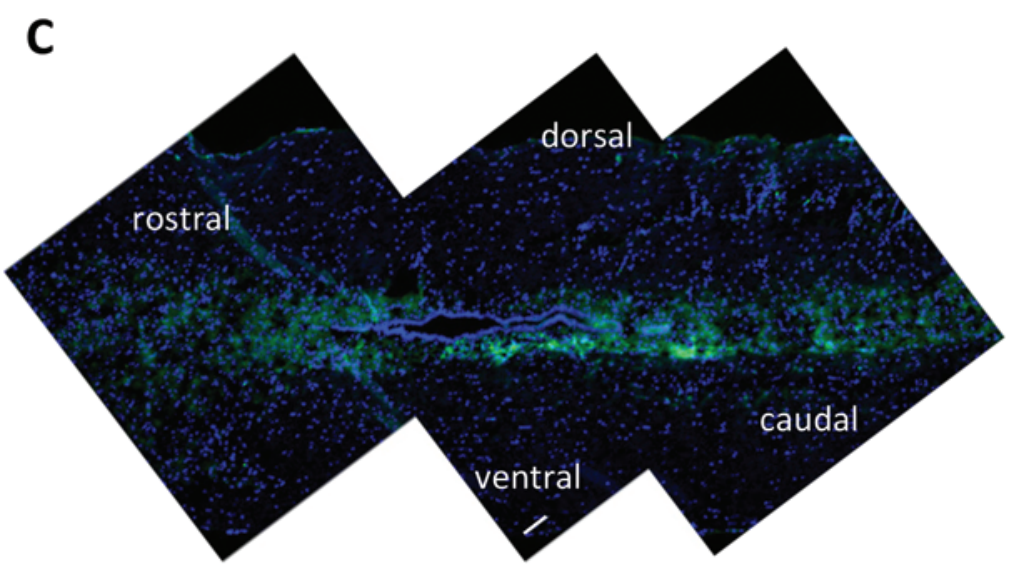

FIG. 3. Photomicrographs and bar graph showing that miR-22 promoted invasion by $1321 \mathrm{~N} 1$ cells in vivo. A: Both control and miR22 expression vector-transfected 1321N1 cells were positive for $\alpha$-tubulin and GFP. The protein level of TIMP2 was downregulated in the miR-22 expression vector group. B and C: Sagittal sections of spinal cord obtained during immunohistochemical analysis confirmed the presence of GFP-positive cells in both groups 1 week after transplantation. D: The extent of the longitudinal direction of GFP-positive grafted cells following transfection with the control vector or miR-22 expression vector was $57.0 \pm 51.4 \mu \mathrm{m}$ and $1041.7 \pm 181.6 \mu \mathrm{m}$, respectively. ${ }^{*} p<0.05$. Bar $=50 \mu \mathrm{m}(A), 100 \mu \mathrm{m}(B$ and $C)$. Figure is available in color online only.

the access of substrates to the catalytic site, whereas the C-terminal domain of TIMP1 and TIMP2 binds to the hemopexin-like domain of pro-MMP-9 and pro-MMP-2, respectively. ${ }^{4}$ Binding of the C-terminal domain is essential for cell surface activation of MMP-2 by MMP-14, ${ }^{14,33}$ and TIMP-2 may play a role in the degradation of the ECM and regulation of glioma invasion. ${ }^{23}$

A correlation between miRNA and glioma invasion has been reported, with some miRNAs being involved in the invasion by regulating the degradation of the ECM. Whereas miR-21 promotes glioma invasiveness by targeting MMP inhibitors, ${ }^{5}$ miR-132 inhibits glioma cell invasion by targeting MMP16, ${ }^{31}$ and miR-23a promotes invasion by modulating MMP-14. ${ }^{7}$ In a recent study, miR-22 inhibited cell proliferation, migration, and invasion via targeting of the $3^{\prime}$-UTR of SIRT1 in the progression of glioblastoma. ${ }^{2}$ Our study suggests a regulatory mechanism by which miR-22 promotes glioma invasion by downregulating TIMP2, which further regulates MMP2 and MMP14.

\section{Conclusions}

Our data suggest that miR-22 acts to regulate the invasion of $1321 \mathrm{~N} 1$ astrocytoma cells by targeting TIMP2 expression. However, this study is preliminary, and future studies with more cases and cell lines are required to fully elucidate our findings. We need to continue investigating miR-22 as a novel treatment target for spinal DAs.

\section{Acknowledgments}

We thank Y. Eguchi for the generous provision of laboratory equipment, and Y. Takahashi for administrative assistance.

\section{References}

1. Buckner JC, Shaw EG, Pugh SL, Chakravarti A, Gilbert MR, Barger GR, et al: Radiation plus procarbazine, CCNU, and vincristine in low-grade glioma. N Engl J Med 374:13441355, 2016

2. Chen H, Lu Q, Fei X, Shen L, Jiang D, Dai D: miR-22 in- 
hibits the proliferation, motility, and invasion of human glioblastoma cells by directly targeting SIRT1. Tumour Biol 37:6761-6768, 2016

3. Cooper PR, Epstein F: Radical resection of intramedullary spinal cord tumors in adults. Recent experience in 29 patients. J Neurosurg 63:492-499, 1985

4. DeClerck YA, Perez N, Shimada H, Boone TC, Langley KE, Taylor SM: Inhibition of invasion and metastasis in cells transfected with an inhibitor of metalloproteinases. Cancer Res 52:701-708, 1992

5. Gabriely G, Wurdinger T, Kesari S, Esau CC, Burchard J, Linsley PS, et al: MicroRNA 21 promotes glioma invasion by targeting matrix metalloproteinase regulators. Mol Cell Biol 28:5369-5380, 2008

6. Houten JK, Cooper PR: Spinal cord astrocytomas: presentation, management and outcome. J Neurooncol 47:219-224, 2000

7. Hu X, Chen D, Cui Y, Li Z, Huang J: Targeting microRNA23 a to inhibit glioma cell invasion via HOXD10. Sci Rep 3:3423, 2013

8. Iorio MV, Croce CM: MicroRNA dysregulation in cancer: diagnostics, monitoring and therapeutics. A comprehensive review. EMBO Mol Med 4:143-159, 2012

9. Jovicic A, Zaldivar Jolissaint JF, Moser R, Silva Santos MDF, Luthi-Carter R: MicroRNA-22 (miR-22) overexpression is neuroprotective via general anti-apoptotic effects and may also target specific Huntington's disease-related mechanisms. PLoS One 8:e54222, 2013

10. Kim MS, Chung CK, Choe G, Kim IH, Kim HJ: Intramedullary spinal cord astrocytoma in adults: postoperative outcome. J Neurooncol 52:85-94, 2001

11. Kleihues P, Soylemezoglu F, Schäuble B, Scheithauer BW, Burger PC: Histopathology, classification, and grading of gliomas. Glia 15:211-221, 1995

12. Kong LM, Liao CG, Zhang Y, Xu J, Li Y, Huang W, et al: A regulatory loop involving miR-22, Sp1, and c-Myc modulates CD147 expression in breast cancer invasion and metastasis. Cancer Res 74:3764-3778, 2014

13. Krol J, Loedige I, Filipowicz W: The widespread regulation of microRNA biogenesis, function and decay. Nat Rev Genet 11:597-610, 2010

14. Li H, Lindenmeyer F, Grenet C, Opolon P, Menashi S, Soria $\mathrm{C}$, et al: AdTIMP-2 inhibits tumor growth, angiogenesis, and metastasis, and prolongs survival in mice. Hum Gene Ther 12:515-526, 2001

15. Minehan KJ, Shaw EG, Scheithauer BW, Davis DL, Onofrio BM: Spinal cord astrocytoma: pathological and treatment considerations. J Neurosurg 83:590-595, 1995

16. Naganuma F, Yoshikawa T, Nakamura T, Iida T, Harada R, Mohsen AS, et al: Predominant role of plasma membrane monoamine transporters in monoamine transport in $1321 \mathrm{~N} 1$, a human astrocytoma-derived cell line. J Neurochem 129:591-601, 2014

17. Nakano A, Tani E, Miyazaki K, Yamamoto Y, Furuyama J: Matrix metalloproteinases and tissue inhibitors of metalloproteinases in human gliomas. J Neurosurg 83:298-307, 1995

18. Ohgaki H, Kleihues P: Epidemiology and etiology of gliomas. Acta Neuropathol 109:93-108, 2005

19. Ohnishi Y, Iwatsuki K, Ishihara M, Shikina T, Shinzawa K, Moriwaki T, et al: Isolation of human adult olfactory sphere cells as a cell source of neural progenitors. Stem Cell Res (Amst) 15:23-29, 2015

20. Ohnishi Y, Iwatsuki K, Shinzawa K, Ishihara M, Moriwaki T, Umegaki M, et al: Adult olfactory sphere cells are a source of oligodendrocyte and Schwann cell progenitors. Stem Cell Res (Amst) 11:1178-1190, 2013

21. Ohnishi Y, Maruo T, Shinzawa K, Iwatsuki K, Moriwaki T, Oshino S, et al: Olfactory sphere cells are a cell source for $\gamma$-aminobutyric acid-producing neurons. J Neurosci Res 93:1293-1304, 2015

22. Raco A, Esposito V, Lenzi J, Piccirilli M, Delfini R, Cantore $\mathrm{G}$ : Long-term follow-up of intramedullary spinal cord tumors: a series of 202 cases. Neurosurgery 56:972-981, 2005

23. Remacle AG, Shiryaev SA, Radichev IA, Rozanov DV, Stec B, Strongin AY: Dynamic interdomain interactions contribute to the inhibition of matrix metalloproteinases by tissue inhibitors of metalloproteinases. J Biol Chem 286:21002-21012, 2011

24. Sato F, Hatano E, Kitamura K, Myomoto A, Fujiwara T, Takizawa S, et al: MicroRNA profile predicts recurrence after resection in patients with hepatocellular carcinoma within the Milan Criteria. PLoS One 6:e16435, 2011

25. Sato F, Tsuchiya S, Terasawa K, Tsujimoto G: Intra-platform repeatability and inter-platform comparability of microRNA microarray technology. PLoS One 4:e5540, 2009

26. Song SJ, Ito K, Ala U, Kats L, Webster K, Sun SM, et al: The oncogenic microRNA miR-22 targets the TET2 tumor suppressor to promote hematopoietic stem cell self-renewal and transformation. Cell Stem Cell 13:87-101, 2013

27. Song SJ, Poliseno L, Song MS, Ala U, Webster K, Ng C, et al: MicroRNA-antagonism regulates breast cancer stemness and metastasis via TET-family-dependent chromatin remodeling. Cell 154:311-324, 2013

28. Tang Y, Liu X, Su B, Zhang Z, Zeng X, Lei Y, et al: microRNA-22 acts as a metastasis suppressor by targeting metadherin in gastric cancer. Mol Med Rep 11:454-460, 2015

29. Tsuchiya N, Izumiya M, Ogata-Kawata H, Okamoto K, Fujiwara Y, Nakai M, et al: Tumor suppressor miR-22 determines p53-dependent cellular fate through post-transcriptional regulation of p21. Cancer Res 71:4628-4639, 2011

30. VanMeter TE, Rooprai HK, Kibble MM, Fillmore HL, Broaddus WC, Pilkington GJ: The role of matrix metalloproteinase genes in glioma invasion: co-dependent and interactive proteolysis. J Neurooncol 53:213-235, 2001

31. Wang H, Li XT, Wu C, Wu ZW, Li YY, Yang TQ, et al: miR132 can inhibit glioma cells invasion and migration by target MMP16 in vitro. Onco Targets Ther 8:3211-3218, 2015

32. Xu D, Takeshita F, Hino Y, Fukunaga S, Kudo Y, Tamaki A, et al: miR-22 represses cancer progression by inducing cellular senescence. J Cell Biol 193:409-424, 2011

33. Zarrabi K, Dufour A, Li J, Kuscu C, Pulkoski-Gross A, Zhi J, et al: Inhibition of matrix metalloproteinase 14 (MMP-14)mediated cancer cell migration. J Biol Chem 286:3316733177,2011

\section{Disclosures}

The authors report no conflict of interest concerning the materials or methods used in this study or the findings specified in this paper.

\section{Author Contributions}

Conception and design: Ohnishi. Acquisition of data: Ohnishi. Analysis and interpretation of data: Ohnishi. Drafting the article: Ohnishi. Critically revising the article: Ohnishi, Fujimoto. Reviewed submitted version of manuscript: Ohnishi, Iwatsuki, Ohkawa, Kinoshita, Shinzawa, Fujimoto, Yoshimine. Approved the final version of the manuscript on behalf of all authors: Ohnishi. Statistical analysis: Ohnishi. Administrative/technical/material support: Iwatsuki, Ishihara, Ohkawa, Kinoshita, Shinzawa, Fujimoto, Yoshimine. Study supervision: Ohnishi, Yoshimine.

\section{Correspondence}

Yu-ichiro Ohnishi, Department of Neurosurgery, Osaka University Medical School, 2-2 Yamadaoka, Suita, Osaka 565-0871, Japan.email: ohnishi@nsurg.med.osaka-u.ac.jp. 